\title{
To the Properties of the Solutions of a Cross-diffusion Parabolic System not in Divergence Form
}

\author{
Mirsaid Aripov, Alisher Matyakubov ${ }^{*}$ \\ Department of Applied Mathematics and Computer Analysis, National University of Uzbekistan, Tashkent, Uzbekistan
}

Copyright $\subset 2017$ by authors, all rights reserved. Authors agree that this article remains permanently open access under the terms of the Creative Commons Attribution License 4.0 International License

\begin{abstract}
The Zeldovich-Barenblatt type solution of the Cauchy problem for a cross-diffusion parabolic system not in divergence form with a source and a variable density is obtained. Based on comparison method the property of finite speed perturbation of distribution is considered. An asymptotic behavior of self-similar solutions, both for slow and fast diffusion cases, is established. It is obtained the system of the nonlinear algebraic equations with the coefficients of the main terms of the asymptotical solution.
\end{abstract}

Keywords Cross-diffusive System, Not in Divergence Form, Finite Speed, Perturbation, Asymptotic Behavior, Numerical Analysis

\section{Introduction}

In this paper we consider the cross-diffusive system of equations not in divergence form

$$
\begin{aligned}
& |x|^{n} \frac{\partial u}{\partial t}=v^{\alpha_{1}} \nabla\left(u^{m_{1}-1} \nabla u\right)+|x|^{n} u^{\beta_{1},}, \\
& |x|^{n} \frac{\partial v}{\partial t}=u^{\alpha_{2}} \nabla\left(v^{m_{2}-1} \nabla v\right)+|x|^{n}{ }_{v}^{\beta_{2}} .
\end{aligned}
$$

in $\mathrm{Q}=\left\{(\mathrm{t}, \mathrm{x}): \mathrm{t}>0, \mathrm{x} \in \mathrm{R}^{\mathrm{N}}\right\}$ with Cauchy conditions

$$
u(0, x)=u_{0}(x), v(0, x)=v_{0}(x),{ }_{x \in R^{N}},
$$

where $\mathrm{n}, \mathrm{m}_{1}, \mathrm{~m}_{2}, \alpha_{1}, \alpha_{2}, \beta_{1}, \beta_{2}$ are given numerical parameters, $\quad \nabla(\cdot)=\operatorname{grad}_{x}(\cdot), \quad u=u(t, x) \geq 0$, $v=v(t, x) \geq 0$ in $Q$.

The system (1) is degenerate. Therefore the system (1) may have no classical solution in the domain at $u=0, v=0, \nabla u=0$, or $\nabla v=0$. In this case we consider weak solution with assumptions $u(t, x), v(t, x) \geq 0$; $v^{\alpha} \nabla\left(u^{m_{1}-1} \nabla u\right) \in C(Q), u^{\alpha_{2}} \nabla\left(v^{m_{1}-1} \nabla v\right) \in C(Q)$ and which satisfies the system (1) in the sense of the distribution [6].

The equations and system of equations not in divergence form are often used to describe various physical phenomena, such as the diffusive processes for biological species, the resistive diffusion phenomena in force-free magnetic fields, curve shortening flow, spreading of infectious diseases and so on (see [1-5]).

The equations not in divergence form different from the classical divergence form equations are more close to the actual processes. For instance, the diffusion for the biological species of divergence form implies that the species is able to move to all locations within its environment with equal probability. But, if we consider this problem with the objective conditions, the population density will affect the rate of diffusion, so a kind of 'biased' diffusion equation will be more realistic, for the non-divergence form diffusion. The diffusion rate is regulated by population density, increasing for large populations and decreasing for small populations.

In [1] for the system (1) for the case $n=0, m_{1}=m_{2}=1$ the authors proved that: (i) when $\min \{a, b\} \leq \lambda_{1}$, where $\lambda_{1}$ is the first eigenvalue of $\Delta$ in $\Omega$ with homogeneous Dirichlet boundary condition, then there exists global positive classical solution, and all positive classical solutions cannot blow up in finite time in the meaning of maximum norm; (ii) when $\min \{a, b\}>\lambda_{1}$, there is no global positive classical solutions. And in addition, if the initial datum $\left(\mathrm{u}_{0}, \mathrm{v}_{0}\right)$ satisfies some assumptions then the positive classical solution is unique and blows up in finite time.

In [2] H. Lu deals with positive solutions of some degenerate and quasilinear parabolic systems not in divergence form

$$
\begin{aligned}
& u_{i t}=f_{i}\left(u_{i+1}\right)\left(\Delta u_{i}+a_{i} u_{i}\right), x \in \Omega, t>0, i=1,2, \ldots, n-1, \\
& u_{n t}=f_{n}\left(u_{1}\right)\left(\Delta u_{n}+a_{n} u_{n}\right), x \in \Omega, t>0 .
\end{aligned}
$$

On some restrictions to the functions $f_{i}\left(u_{i+1}\right), i=1,2, \ldots, n-1$, he proved the local existence and uniqueness of classical solution. Moreover, it was proven 
that: if $\min \left\{a_{1}, \ldots, a_{n}\right\} \leq \lambda_{1}$ there exists global positive classical solution, and all positive classical solutions cannot blow up in finite time in the meaning of maximum norm. Also, in this reference it is shown that if $\min \left\{\mathrm{a}_{1}, \ldots, \mathrm{a}_{\mathrm{n}}\right\}>\lambda_{1}$, and the initial datum $\left(\mathrm{u}_{10}, \ldots, \mathrm{u}_{\mathrm{n} 0}\right)$ satisfies to some assumptions, then the positive classical solution is unique and blows up in finite time.

Some properties of solutions for the non-divergence form, for a single equation, such as the existence, non-uniqueness, the blow-up properties etc. have been discussed by many authors [3-5]. For example in [3] the existence of a unique viscosity solution of the Cauchy problem (1)-(2) at $n=0, m_{1}=m_{2}=1$ was proved. In the work [4], self-similar solutions in the form of

$u(t, x)=(t+1)^{-\alpha} f\left((t+1)^{\beta}|x|^{2}\right)$ were studied. The existence and uniqueness of solutions with compact support, which implies that the self-similar solution is shrinking, is proved. The convergent rates of these solutions on the boundary of the support are established. The convergent speeds of the solutions are considered, with Dirac function at $\mathrm{t} \rightarrow \infty$. In reference [5] it is studied the asymptotic behavior of solutions at $\mathrm{t} \rightarrow \infty$, and investigated slow and fast diffusion cases.

Finite speed perturbation of distribution (FSPD) property and asymptotic behavior of self-similar solutions for divergence systems are considered in $[8-9,12,13]$.

In references [10] and [11], it is studied the Cauchy problem for the following two equations with variable coefficients

$$
\rho(x) \frac{\partial u}{\partial t}=\operatorname{div}\left(u^{m-1}|\nabla u|^{p-2} \nabla u\right)+\rho(x) u^{\beta},
$$

and

$$
\rho(x) \frac{\partial u}{\partial t}=\operatorname{div}\left(u^{m-1}|\nabla u|^{p-2} \nabla u\right)+u^{\beta}, \mathrm{x} \in \mathrm{R}^{N}, t>0 .
$$

where $\mathrm{p}>1, \mathrm{~m}+\mathrm{p}-3>0, \beta>\mathrm{m}+\mathrm{p}-2, \rho(\mathrm{x})=|\mathrm{x}|^{-\mathrm{n}}$ or $\rho(\mathrm{x})=(1+|\mathrm{x}|)^{-\mathrm{n}}$. They showed that under some restrictions to the parameters and initial data, any nontrivial solution to the Cauchy problem blows up in finite time. Moreover, the authors established a sharp universal estimate of the solution near the blow-up point.

This paper is devoted to constructing Zeldovich-Barenblatt type solution to the cross-diffusive system equation (1). And based on comparison method the property a FSPD of the Cauchy problem for a cross-diffusion parabolic system not in divergence form is established. An asymptotic behavior of self-similar solution of a cross-diffusion parabolic system of equations in non-divergence form for slow and the fast diffusion cases (depending on value of the numerical parameters) is discussed. On the basis of this asymptotic of solutions, suitable initial approximations are offered in the iterative process for the slow and fast diffusion cases, depending on the values of the numeric parameters.

\section{The Self-similar System of Equations}

Below we provide a method of nonlinear splitting [1] to construct self-similar equation. For construction of the self-similar solutions of the system (1) we find the solutions $u(t, x), v(t, x)$ in the form

$$
\begin{aligned}
& \mathrm{u}(\mathrm{t}, \mathrm{x})=\overline{\mathrm{u}}(\mathrm{t}) \mathrm{w}(\tau(\mathrm{t}), \mathrm{r}) \\
& \mathrm{v}(\mathrm{t}, \mathrm{x})=\overline{\mathrm{v}}(\mathrm{t}) \psi(\tau(\mathrm{t}), \mathrm{r})
\end{aligned}
$$

Here $r=|x|$.

Let $\overline{\mathrm{u}}(\mathrm{t}), \overline{\mathrm{v}}(\mathrm{t})$ be in the form

$$
\overline{\mathrm{u}}(\mathrm{t})=(\mathrm{T}+\mathrm{t})^{1 /\left(1-\beta_{1}\right)}, \overline{\mathrm{v}}(\mathrm{t})=(\mathrm{T}+\mathrm{t})^{1 /\left(1-\beta_{2}\right)} .
$$

Using (3) the system (1) can be reduced to the following form

$$
\begin{aligned}
& \mathrm{r}^{\mathrm{n}} \frac{\partial \mathrm{w}}{\partial \tau}=\mathrm{d}_{1} \psi^{\alpha} 1^{1-\mathrm{N}} \frac{\partial}{\partial \mathrm{r}}\left(\mathrm{r}^{\mathrm{N}-1} \mathrm{w}^{-1} \frac{\partial \mathrm{w}}{\partial \mathrm{r}}\right)+ \\
& +b_{1}{ }^{n} \tau^{-1}\left({ }_{w} \beta_{1}-w\right), \\
& \mathrm{r}^{\mathrm{n}} \frac{\partial \psi}{\partial \tau}=\mathrm{d}_{2} \mathrm{w}^{\alpha} \mathrm{r}^{1-\mathrm{N}} \frac{\partial}{\partial \mathrm{r}}\left(\mathrm{r}^{\mathrm{N}-1} \psi^{\mathrm{m}_{2}-1} \frac{\partial \psi}{\partial \mathrm{r}}\right)+ \\
& +\mathrm{b}_{2} \mathrm{r}^{\mathrm{n}} \tau^{-1}\left(\psi^{\left.\beta_{2}-\psi\right)}\right. \text {, }
\end{aligned}
$$

where

$$
\begin{gathered}
\tau(\mathrm{t})=\int(\mathrm{T}+\mathrm{t}) \frac{\alpha_{1}}{1-\beta_{2}}+\frac{\mathrm{m}_{1}-1}{1-\beta_{1}} \text { dt at } \frac{\alpha_{1}}{1-\beta_{2}}+\frac{\mathrm{m}_{1}-1}{1-\beta_{1}}+1 \neq 0, \\
\tau(\mathrm{t})=\ln (\mathrm{T}+\mathrm{t}) \text { at } \frac{\alpha_{1}}{1-\beta_{2}}+\frac{\mathrm{m}_{1}-1}{1-\beta_{1}}+1=0, \\
\mathrm{~b}_{\mathrm{i}}=\frac{1}{1-\beta_{\mathrm{i}}} \cdot \frac{1}{\frac{\alpha_{\mathrm{i}}}{1-\beta_{3}-\mathrm{i}}+\frac{\mathrm{m}_{\mathrm{i}}-1}{1-\beta_{\mathrm{i}}}+1}, \mathrm{i}=1,2 . \mathrm{r}=|\mathrm{x}| . \\
\frac{\alpha_{1}}{1-\beta_{2}}+\frac{\mathrm{m}_{1}-1}{1-\beta_{1}}=\frac{\alpha_{2}}{1-\beta_{1}}+\frac{\mathrm{m}_{2}-1}{1-\beta_{2}} .
\end{gathered}
$$

It is easy to establish that the system (4) has the self-similar solution

$$
\mathrm{w}(\tau, \mathrm{r})=\mathrm{f}(\xi), \psi(\tau, \mathrm{r})=\phi(\xi), \xi=\mathrm{r} \tau^{-\frac{1}{\mathrm{n}+2}}
$$

where $\xi$ is the self-similar variable and the functions $f(\xi), \phi(\xi)$ satisfies the following self-similar system of equations 


$$
\begin{aligned}
& \phi^{\alpha_{1}} \xi^{1-N} \frac{d}{d \xi}\left(\xi^{N-1} \mathrm{f}_{1}^{-1} \frac{d f}{d \xi}\right)+ \\
& +\frac{1}{n+2} \xi^{n+1} \frac{d f}{d \xi}+b_{1} \xi^{n}\left(f^{\left.\beta_{1}-f\right)}=0,\right. \\
& f^{\alpha} \xi^{1-N} \frac{d}{d \xi}\left(\xi^{N-1} \phi^{m_{2}-1} \frac{d \phi}{d \xi}\right)+ \\
& +\frac{1}{n+2} \xi^{n+1} \frac{d \phi}{d \xi}+b_{2} \xi^{n}\left(\phi^{\beta}-\phi\right)=0 .
\end{aligned}
$$

According to the statement of the original problem we will consider nontrivial, nonnegative solutions of the system (6) satisfying the following conditions

$$
\begin{aligned}
& \mathrm{f}(0)=\mathrm{M}_{1}, \phi(0)=\mathrm{M}_{2}, \mathrm{M}_{1} \in \mathrm{R}^{+}, \mathrm{M}_{2} \in \mathrm{R}^{+}, \\
& \mathrm{f}\left(\mathrm{d}_{1}\right)=\phi\left(\mathrm{d}_{2}\right)=0,0<\mathrm{d}_{1}<\infty, 0<\mathrm{d}_{2}<\infty .
\end{aligned}
$$

\section{Slow Diffusion (case $m_{3-i}>1+\alpha_{i}$, $\mathrm{i}=1,2$ ).}

Applying the method of comparison of solutions [6] and method of standard equations [7] for solving the problem (6)-(7) we obtain the estimates for the solution of the problem (1)-(2).

It should be noted, that the functions

$$
\overline{\mathrm{f}}(\xi)=\mathrm{A}\left(\mathrm{a}-\xi^{\mathrm{n}+2}\right)_{+}^{\mathrm{p}_{1}}, \bar{\phi}(\xi)=\mathrm{B}\left(\mathrm{a}-\xi^{\mathrm{n}+2}\right)_{+}^{\mathrm{p}_{2}},
$$

where

$$
p_{i}=\frac{m_{3-i}-\alpha_{i}-1}{\left(m_{1}-1\right)\left(m_{2}-1\right)-\alpha_{1} \alpha_{2}}, i=1,2 \text {, }
$$

$(\mathrm{b})_{+}=\max (0, \mathrm{~b}), \quad \mathrm{a}^{\mathrm{p}_{1}} \mathrm{~A}=\mathrm{M}_{1}, \quad \mathrm{a}^{\mathrm{p}} \mathrm{p}_{2} \mathrm{~B}=\mathrm{M}_{2}$

$\xi<\mathrm{a}^{1 /(\mathrm{n}+2)}$ satisfy the conditions (7).

Theorem 1. Let $m_{3-i}>1+\alpha_{i}, i=1,2$,

$$
\begin{gathered}
M_{i}^{\beta_{i}-1} \leq 1+\frac{N+n}{n+2} \frac{p_{i}}{b_{i}\left(p_{i} m_{i}-1\right)}, \quad i=1,2, \\
M_{i}^{m_{i}-1} M_{3-i}^{\alpha_{i}}=\frac{a}{\left(p_{i} m_{i}-1\right)(n+2)^{2}}, i=1,2, \\
u_{+}(0, x) \geq u_{0}(x), v_{+}(0, x) \geq v_{0}(x), \quad x \in R^{N} .
\end{gathered}
$$

Then the problem (1) - (2) has a global solution, which satisfies estimates

$$
\begin{aligned}
& \mathrm{u}(\mathrm{t}, \mathrm{x}) \leq \mathrm{u}_{+}(\mathrm{t}, \mathrm{x})=\overline{\mathrm{u}}(\mathrm{t}) \overline{\mathrm{f}}(\xi), \\
& \mathrm{v}(\mathrm{t}, \mathrm{x}) \leq \mathrm{v}_{+}(\mathrm{t}, \mathrm{x})=\overline{\mathrm{v}}(\mathrm{t}) \bar{\phi}(\xi),
\end{aligned}
$$

in $\mathrm{Q}$.

Proof. To prove the theorem we use the method of comparison of solutions. As comparable functions we take the $u_{+}(t, x), v_{+}(t, x)$, that defined by (9). Then, according to (3), (5) and

$$
\mathrm{M}_{\mathrm{i}}^{\mathrm{m}_{\mathrm{i}}-1} \mathrm{M}_{3-\mathrm{i}}^{\alpha_{\mathrm{i}}}=\frac{\mathrm{a}}{\left(\mathrm{p}_{\mathrm{i}} \mathrm{m}_{\mathrm{i}}-1\right)(\mathrm{n}+2)^{2}}, \mathrm{i}=1,2,
$$

we obtain

$$
\begin{array}{r}
\mathrm{Lu}_{+}(\mathrm{t}, \mathrm{x})=\left(-\frac{\mathrm{p}_{1}(\mathrm{~N}+\mathrm{n})}{(\mathrm{n}+2)\left(\mathrm{p}_{1} \mathrm{~m}_{1}-1\right)}-\mathrm{b}_{1}\right) \xi^{\mathrm{n}} \overline{\mathrm{f}}(\xi)+ \\
+\mathrm{b}_{1} \xi^{\mathrm{n}} \overline{\mathrm{f}}^{\beta_{1}}(\xi), \\
\mathrm{Lv}_{+}(\mathrm{t}, \mathrm{x})=\left(-\frac{\mathrm{p}_{2}(\mathrm{~N}+\mathrm{n})}{(\mathrm{n}+2)\left(\mathrm{p}_{2} \mathrm{~m}_{2}-1\right)}-\mathrm{b}_{2}\right) \xi^{\mathrm{n}} \bar{\phi}(\xi)+ \\
+\mathrm{b}_{2} \xi^{\mathrm{n}}-\beta_{2}(\xi)
\end{array}
$$

From the last expressions and the inequalities $\overline{\mathrm{f}}(\xi) \leq \mathrm{Aa}^{\mathrm{p}} 1=\mathrm{M}_{1}, \bar{\phi}(\xi) \leq \mathrm{Ba}^{\mathrm{p}} 2=\mathrm{M}_{2}$ it follows that to fulfill the inequalities $\mathrm{Lu}_{+} \leq 0, \mathrm{Lv}_{+} \leq 0$ is enough to fulfill the conditions

$$
M_{i}^{\beta_{i}-1} \leq 1+\frac{N+n}{n+2} \frac{p_{i}}{b_{i}\left(p_{i} m_{i}-1\right)}, \quad i=1,2 .
$$

These inequalities follow from the conditions of the theorem. Then, by the comparison theorem of solutions there is a global solution in Q for the problem (1)-(2) with the following estimates

$$
\mathrm{u}_{+}(\mathrm{t}, \mathrm{x}) \geq \mathrm{u}(\mathrm{t}, \mathrm{x}), \mathrm{v}_{+}(\mathrm{t}, \mathrm{x}) \geq \mathrm{v}(\mathrm{t}, \mathrm{x}), \mathrm{x} \in \mathrm{R}^{\mathrm{N}}
$$

The proof of the theorem is completed.

Thus, we obtained FSPD property of the solutions of the Cauchy problem (1), (2).

\section{The Asymptotic Behavior of Self-similar Solutions of the Problem (6)-(7)}

Let's use the notations:

$$
\begin{aligned}
& b_{i 1}=p_{i}\left(p_{i} m_{i}-1\right), b_{i 2}=-\frac{p_{i}}{(n+2)^{2}}, \\
& b_{i 3}=\frac{b_{i}}{(n+2)^{2} a}, \quad i=1,2 .
\end{aligned}
$$

Assume $\frac{\alpha_{1}}{1-\beta_{2}}+\frac{m_{1}-1}{1-\beta_{1}}=\frac{\alpha_{2}}{1-\beta_{1}}+\frac{m_{2}-1}{1-\beta_{2}}$. Then the following theorem is valid.

Theorem 2. Let $\frac{\alpha_{1}}{1-\beta_{2}}+\frac{m_{1}-1}{1-\beta_{1}}+1>0$. Then compactly supported solution of the problem (6), (7) has the following asymptotic behavior 


$$
\begin{aligned}
& f(\xi)=c_{1}\left(a-\xi^{n+2}\right)^{p_{1}}(1+o(1)), \\
& \phi(\xi)=c_{2}\left(a-\xi^{n+2}\right)^{p_{2}}(1+o(1)),
\end{aligned}
$$

at $\xi \rightarrow a^{\frac{1}{n+2}}$, where the parameters $\mathrm{p}_{\mathrm{i}}, \mathrm{i}=1,2$ satisfy one of the following conditions

1. $\mathrm{p}_{1}=\frac{1}{1-\beta_{1}}, \mathrm{p}_{2}=\frac{1}{1-\beta_{2}}$ and the coefficients $\mathrm{c}_{\mathrm{i}}$ $(i=1,2)$ are solution of the systems of nonlinear algebraic equations

$$
\mathrm{b}_{\mathrm{i} 1} \mathrm{c}_{\mathrm{i}}^{\mathrm{m}_{\mathrm{i}}-1} \mathrm{c}_{3-\mathrm{i}}^{\alpha_{\mathrm{i}}}+\mathrm{b}_{\mathrm{i} 2}+\mathrm{b}_{\mathrm{i} 3} \mathrm{c}_{\mathrm{i}}^{\beta_{\mathrm{i}}-1}=0, \mathrm{i}=1,2 .
$$

2. $\mathrm{p}_{\mathrm{i}}<\frac{1}{1-\beta_{\mathrm{i}}}, \mathrm{p}_{\mathrm{i}} \mathrm{m}_{\mathrm{i}}>1, \mathrm{i}=1,2$ and the coefficients $\mathrm{c}_{\mathrm{i}}$ $(i=1,2)$ are solution of the systems of nonlinear algebraic equations

$$
b_{i 1} c_{i}^{m_{i}-1} c_{3-i}^{\alpha_{i}}+b_{i 2}=0, i=1,2
$$

3. $\mathrm{p}_{1}=\frac{1}{1-\beta_{1}}, \mathrm{p}_{2}<\frac{1}{1-\beta_{2}}, \mathrm{p}_{2} \mathrm{~m}_{2}>1$ and the coefficients $c_{i}(i=1,2)$ are solution of the systems of nonlinear algebraic equations

$$
\left\{\begin{array}{l}
b_{11} c_{1}^{m_{1}-1} c_{2}^{\alpha_{1}}+b_{12}+b_{13} c_{1}^{\beta_{1}-1}=0 \\
b_{21} c_{2}^{m_{2}-1} c_{1}^{\alpha_{2}}+b_{22}=0
\end{array}\right.
$$

4. $\mathrm{p}_{1}<\frac{1}{1-\beta_{1}}, \mathrm{p}_{2}=\frac{1}{1-\beta_{2}}, \mathrm{p}_{1} \mathrm{~m}_{1}>1 \quad$ and the coefficients $c_{i}(i=1,2)$ are solution of the systems of nonlinear algebraic equations

$$
\left\{\begin{array}{l}
b_{11} c_{1}^{m_{1}-1} c_{2}^{\alpha_{1}}+b_{12}=0, \\
b_{21} c_{2}^{m_{2}-1} c_{1}^{\alpha_{2}}+b_{22}+b_{23} c_{2}^{\beta_{2}-1}=0 .
\end{array}\right.
$$

Proof. We transform the system (6)-(7) into the relatively easy form. Let for investigation

$$
\begin{aligned}
& \mathrm{f}(\xi)=\overline{\mathrm{f}}(\xi) \mathrm{y}_{1}(\eta), \phi(\xi)=\bar{\phi}(\xi) \mathrm{y}_{2}(\eta), \\
& \eta=-\ln \left(\mathrm{a}-\xi^{\mathrm{n}+2}\right)
\end{aligned}
$$

where $\quad \overline{\mathrm{f}}(\xi)=\left(\mathrm{a}-\xi^{\mathrm{n}+2}\right)^{\mathrm{p}_{1}}, \bar{\phi}(\xi)=\left(\mathrm{a}-\xi^{\mathrm{n}+2}\right)^{\mathrm{p}_{2}}$, $\mathrm{y}_{1}(\eta), \mathrm{y}_{2}(\eta)$ are unknown functions.

Now let us investigate the asymptotic behavior of solutions of the system (6) at $\xi \rightarrow a^{\frac{1}{n+2}}$.

After the transformation (11) the system (6) will take the following form

$$
\begin{aligned}
& y_{3-i}^{\alpha_{i}} \frac{d}{d \eta}\left(L y_{i}\right)+a_{i 2}(\eta)\left(\frac{d y_{i}}{d \eta}+a_{i 0}(\eta) y_{i}\right)+ \\
& +a_{i 1}(\eta) y_{3-i}^{\alpha} \alpha_{i}+a_{i 3}(\eta) y_{i}+a_{i 4}(\eta) y_{i}^{\beta_{i}}=0,
\end{aligned}
$$

$(i=1,2)$.

Here $L y_{i}=y_{i}^{m_{i}}-1\left(\frac{d y_{i}}{d \eta}+a_{i 0}(\eta) y_{i}\right), \quad a_{i 0}(\eta)=-p_{i}$, $a_{i 1}(\eta)=\frac{n+N}{n+2} \frac{e^{-\eta}}{a-e^{-\eta}}-p_{i} m_{i}+1, \quad a_{i 2}(\eta)=\frac{1}{(n+2)^{2}}$,

$$
a_{i 3}(\eta)=-\frac{b_{i}}{(n+2)^{2}} \frac{e^{-\eta}}{a-e^{-\eta}}
$$

$a_{i 4}(\eta)=\frac{b_{i}}{(n+2)^{2}} \frac{e^{-\left(1+p_{i}\left(\beta_{i}-1\right)\right) \eta}}{a-e^{-\eta}}, i=1,2$.

It is supposed that $\xi \in\left[\xi_{0}, \xi_{1}\right), \quad 0<\xi_{0}<\xi_{1}$, $\xi_{1}=a^{\frac{1}{n+2}}$.

Therefore, the function $\boldsymbol{\eta}(\boldsymbol{\xi})$ has the properties:

$$
\begin{aligned}
& \eta^{\prime}(\xi)>0 \quad \text { at } \quad \xi \in\left[\xi_{0}, \xi_{1}\right) \text {, } \\
& \eta \mathrm{\eta}=\boldsymbol{\eta}\left(\xi_{0}\right)>0, \lim _{\xi \longrightarrow \xi_{1}} \eta(\xi)=+\infty \text {. }
\end{aligned}
$$

It follows from self-similar system of equations (12) the following limitations

$$
\lim _{\eta \rightarrow+\infty} a_{i j}(\eta)=a_{i j}^{0} \quad(i=1,2 ; j=0,1,2,3,4)
$$

are exist, and finite and nonzero, that is $0<\left|\mathrm{a}_{\mathrm{ij}}^{0}\right|<+\infty$.

Further, we consider the solutions of the system (12), in a certain neighborhood $+\infty$ satisfies the inequalities

$$
y_{i}(\eta)>0, \quad y_{i}^{\prime}+a_{i 0}(\eta) y_{i} \neq 0 \quad(i=1,2) .
$$

Assuming

$$
v_{i}(\eta)=y_{i} m_{i}-1\left(\frac{d y_{i}}{d \eta}+a_{i 0}(\eta) y_{i}\right), \quad i=1,2
$$

in the system (12) we obtain the identity

$$
\begin{aligned}
& \mathrm{v}_{\mathrm{i}}^{\prime}(\eta) \equiv-\mathrm{a}_{\mathrm{i} 1}(\eta) \mathrm{v}_{\mathrm{i}}(\eta)-\mathrm{a}_{\mathrm{i} 2}(\eta) \mathrm{v}_{\mathrm{i}}(\eta) \mathrm{y}_{\mathrm{i}}^{1-\mathrm{m}_{\mathrm{i}}} \mathrm{y}_{3-\mathrm{i}}^{-\alpha_{i}}- \\
& -a_{i 3}(\eta) y_{i} y_{3-i}^{-\alpha_{i}}-a_{i 4}(\eta) y_{i}^{\beta_{i}} y_{3-i}-\alpha_{i}, \quad(i=1,2) \text {. }
\end{aligned}
$$

Now consider the functions

$$
\begin{aligned}
& g_{i}\left(\lambda_{i}, \eta\right) \equiv-a_{i 1}(\eta) \lambda_{i}-a_{i 2}(\eta) \lambda_{i} y_{i}^{1-m_{i}} y_{3-i}^{-\alpha_{i}}- \\
& -a_{i 3}(\eta) y_{i} y_{3-i}^{-\alpha_{i}}-a_{i 4}(\eta) y_{i}^{\beta_{i}} y_{3-i}-\alpha_{i}
\end{aligned}
$$


where $\lambda_{\mathrm{i}} \in \mathrm{R}, \quad(\mathrm{i}=1,2)$.

$$
\begin{aligned}
& \text { Suppose } \frac{\alpha_{1}}{1-\beta_{2}}+\frac{m_{1}-1}{1-\beta_{1}}+1>0 . \\
& \text { Then } \lim _{\eta \rightarrow+\infty} a_{i 2}(\eta)=\frac{1}{(n+2)^{2}}, \\
& \lim _{\eta \rightarrow+\infty} a_{i 1}(\eta)=-p_{i} m_{i}+1, \lim _{\eta \rightarrow+\infty} a_{i 3}(\eta)=0(i=1,2) \\
& \lim _{\eta \rightarrow+\infty} a_{i 4}(\eta)=\left\{\begin{array}{l}
\frac{b_{i}}{(n+2)^{2}}, \text { at } 1+p_{i}\left(\beta_{i}-1\right)=0, \\
0, \text { at } 1+p_{i}\left(\beta_{i}-1\right)>0, \quad i=1,2
\end{array}\right.
\end{aligned}
$$

and the functions $g_{i}\left(\lambda_{i}, \eta\right) \quad(i=1,2)$ preserves sign on some interval $\left[\eta_{1},+\infty\right) \subset\left[\eta_{0},+\infty\right)$ for every fixed value $\lambda_{\mathrm{i}}$ $(\mathrm{i}=1,2)$.

So, the functions $g_{i}\left(\lambda_{i}, \eta\right) \quad(i=1,2)$ for all $\eta \in\left[\eta_{1},+\infty\right)$ satisfies one of the inequalities

$$
g_{i}\left(\lambda_{i}, \eta\right)>0 \text { or } g_{i}\left(\lambda_{i}, \eta\right)<0 \quad(i=1,2) .
$$

Suppose now that for the functions $v_{i}(\eta)(i=1,2)$ limit at $\eta \rightarrow+\infty$ does not exist. Consider the case when one of the inequalities (16) is satisfied. As $v_{i}(\eta)(i=1,2)$ are oscillating functions around $\bar{v}_{i}=\lambda_{i}(i=1,2)$ its graph intersects this straight line infinitely many times in $\left[\eta_{1},+\infty\right)$. But this is impossible, since in the interval $\left[\eta_{1},+\infty\right)$ just one of the inequalities (16) are valid and therefore, from (15) it follows that graph of the function $v_{i}(\eta)(i=1,2)$ intersects the straight line $\bar{v}_{i}=\lambda_{i}(i=1,2)$ only once in the interval $\left[\eta_{1},+\infty\right)$. Accordingly, the function $\mathrm{v}_{\mathrm{i}}(\eta)(\mathrm{i}=1,2)$ has a limit at $\eta \rightarrow+\infty$.

By assumption the functions $v_{i}(\eta)(i=1,2)$ defined in (13), has a limit at $\eta \rightarrow+\infty$. Then $y_{i}^{\prime}(\eta)(i=1,2)$ has a limit at $\eta \rightarrow+\infty$, and this limit is zero. Then

$$
\begin{gathered}
v_{i}(\eta)=y_{i}^{m_{i}-1}\left(\frac{d y_{i}}{d \eta}+a_{i} 0(\eta) y_{i}\right)= \\
=a_{i 0}^{0}\left(y_{i}^{0}\right)^{m_{i}}+o(1), \quad i=1,2
\end{gathered}
$$

at $\eta \rightarrow+\infty$. And by (14) derivatives of functions $v_{i}(\eta)$ $(\mathrm{i}=1,2)$ has a limit at $\eta \rightarrow+\infty$, what are obviously equal to zero.

Consequently, it is necessary

$$
\begin{aligned}
& \lim _{\eta \rightarrow+\infty}\left(a_{i 1}(\eta) v_{i}(\eta)+a_{i 2}(\eta) v_{i}(\eta) y_{i}^{1-m_{i}} y_{3-i}^{-\alpha_{i}}\right)+ \\
& +\lim _{\eta \rightarrow+\infty}\left(a_{i 3}(\eta) y_{i} y_{3-i}-\alpha_{i}+a_{i 4}(\eta) y_{i}^{\beta_{i}} y_{3-i}^{-\alpha_{i}}\right)=0
\end{aligned}
$$

$(i=1,2)$.

From this expression, it is easy to see that the system (12) has a solution $\left(\mathrm{y}_{1}(\eta), \mathrm{y}_{2}(\eta)\right)$ with a finite non-zero limit at $\eta \rightarrow+\infty$, necessary, to compliance with one of the conditions of Theorem 2 .

Then the compactly supported solution of the problem (6)-(7) has an asymptotic of the form (10) as $\xi \rightarrow \frac{1}{\mathrm{n}+2}$.

The theorem 2 is proved.

Corollary 1. A weak solution of the problem (1)-(2) has the following asymptotics

$$
\begin{aligned}
& \mathrm{u}_{\mathrm{A}}(\mathrm{t}, \mathrm{x}) \approx \mathrm{c}_{1}(\mathrm{~T}+\mathrm{t}) \frac{1}{1-\beta_{1}}\left(\mathrm{a}-\frac{|\mathrm{x}|^{\mathrm{n}+2}}{\tau}\right)^{\mathrm{p}_{1}}, \\
& \mathrm{v}_{\mathrm{A}}(\mathrm{t}, \mathrm{x}) \approx \mathrm{c}_{2}(\mathrm{~T}+\mathrm{t}) \frac{1}{1-\beta_{2}}\left(\mathrm{a}-\frac{|\mathrm{x}|^{\mathrm{n}+2}}{\tau}\right)^{\mathrm{p}_{2}},
\end{aligned}
$$

at $|x| \rightarrow a \frac{1}{n+2} \frac{1}{n+2}$, where constants $c_{1}, c_{2}, p_{1}, p_{2}$ defined above.

5. Fast Diffusion (case $p_{i}<0, \quad i=1,2$ ).

Suppose that the problem is satisfies the condition

$$
f^{\prime}(0)=0, \phi^{\prime}(0)=0, f(\infty)=0, \phi(\infty)=0 .
$$

Note that the functions

$$
\begin{aligned}
& \bar{f}(\xi)=\left(\mathrm{a}+\xi^{\mathrm{n}+2}\right)^{\mathrm{p}_{1}}, \bar{\phi}(\xi)=\left(\mathrm{a}+\xi^{\mathrm{n}+2}\right)^{\mathrm{p}_{2}}, \\
& \mathrm{p}_{\mathrm{i}}=\frac{\mathrm{m}_{3-\mathrm{i}}-1-\alpha_{\mathrm{i}}}{\left(\mathrm{m}_{1}-1\right)\left(\mathrm{m}_{2}-1\right)-\alpha_{1} \alpha_{2}}, \quad \mathrm{i}=1,2 .
\end{aligned}
$$

with a $>0$, satisfies the condition of (17).

Theorem 3. Let $p_{i}<0, \beta_{i}<1$,

$$
\begin{gathered}
\mathrm{p}_{\mathrm{i}}(\mathrm{n}+2)(\mathrm{n}+\mathrm{N})-\mathrm{b}_{\mathrm{i}}+\mathrm{b}_{\mathrm{i}} \mathrm{a}_{\mathrm{i}}\left(\beta_{\mathrm{i}}-1\right) \geq 0, \\
\mathrm{p}_{\mathrm{i}}=\frac{1}{\mathrm{~m}_{\mathrm{i}}}\left(1-\frac{1}{(\mathrm{n}+2)^{2}}\right), \mathrm{i}=1,2, \\
\mathrm{u}_{0}(\mathrm{x}) \geq \mathrm{u}_{-}(0, \mathrm{x}), \mathrm{v}_{0}(\mathrm{x}) \geq \mathrm{v}_{-}(0, \mathrm{x}), \mathrm{x} \in \mathrm{R}^{\mathrm{N}} .
\end{gathered}
$$

Then the problem (1) - (2) has a global solution in Q and it satisfies estimation

$$
\mathrm{u}(\mathrm{t}, \mathrm{x}) \geq \mathrm{u}_{-}(\mathrm{t}, \mathrm{x}), \mathrm{v}(\mathrm{t}, \mathrm{x}) \geq \mathrm{v}_{-}(\mathrm{t}, \mathrm{x}), \mathrm{x} \in \mathrm{R},
$$

where 


$$
\mathrm{u}_{-}(\mathrm{t}, \mathrm{x})=\overline{\mathrm{u}}(\mathrm{t}) \overline{\mathrm{f}}(\xi), \quad \mathrm{v}_{-}(\mathrm{t}, \mathrm{x})=\overline{\mathrm{v}}(\mathrm{t}) \bar{\phi}(\xi)
$$

The proof of the Theorem 3 is similar to the proof of the Theorem 1 .

Introduce the notations

$$
b_{i 4}=p_{i}\left(\frac{n+N}{n+2}+p_{i} m_{i}-1\right), b_{i 5}=\frac{p_{i}-b_{i}}{(n+2)^{2}}, \quad i=1,2 .
$$

Assume $\frac{\alpha_{1}}{1-\beta_{2}}+\frac{m_{1}-1}{1-\beta_{1}}=\frac{\alpha_{2}}{1-\beta_{1}}+\frac{m_{2}-1}{1-\beta_{2}}$. Then the following theorem is valid:

Theorem 4. Let $\frac{\alpha_{1}}{1-\beta_{2}}+\frac{m_{1}-1}{1-\beta_{1}}+1>0, \quad \beta_{\mathrm{i}}>1$,

$\mathrm{p}_{\mathrm{i}}<-\frac{\mathrm{N}-2}{\mathrm{~m}_{\mathrm{i}}(\mathrm{n}+2)}, \mathrm{i}=1,2$. Then solution of the problem

(6), (17), vanishing at infinity, has an asymptotic

$$
\mathrm{f}(\xi)=\mathrm{c}_{3}\left(\mathrm{a}+\xi^{\mathrm{n}+2}\right)^{\mathrm{p}_{1}}, \phi(\xi)=\mathrm{c}_{4}\left(\mathrm{a}+\xi^{\mathrm{n}+2}\right)^{\mathrm{p}_{2}},
$$

at $\xi \rightarrow \infty$, where the coefficients $\mathrm{c}_{\mathrm{i}}(\mathrm{i}=3,4)$ are solution of systems of the nonlinear algebraic equations

$$
b_{i 4} c_{i}^{m_{i}-1} c_{3-i}^{\alpha_{i}}+b_{i 5}=0, i=3,4 \text {. }
$$

The proof of the Theorem 4 analogously to the proof of the Theorem 2.

Corollary 2. A weak solution of the problem (1)-(2) has the following asymptotic

$$
\begin{aligned}
& \mathrm{u}_{\mathrm{A}}(\mathrm{t}, \mathrm{x}) \approx \mathrm{c}_{1}(\mathrm{~T}+\mathrm{t}) \frac{1}{1-\beta_{1}}\left(\mathrm{a}+\frac{|\mathrm{x}|^{\mathrm{n}+2}}{\tau}\right)^{\mathrm{p}_{1}}, \\
& \mathrm{v}_{\mathrm{A}}(\mathrm{t}, \mathrm{x}) \approx \mathrm{c}_{2}(\mathrm{~T}+\mathrm{t}) \frac{1}{1-\beta_{2}}\left(\mathrm{a}+\frac{|\mathrm{x}|^{\mathrm{n}+2}}{\tau}\right)^{\mathrm{p}_{2}},
\end{aligned}
$$

at $|\mathrm{x}| \tau^{-\frac{1}{\mathrm{n}+2} \rightarrow+\infty}$, where constants $\mathrm{c}_{1}, \mathrm{c}_{2}, \mathrm{p}_{1}, \mathrm{p}_{2}$ defined above.

\section{Numerical Analysis of Solutions}

We note that due to non-uniqueness of solutions many different cases arise in the numerical study of the problem (1)-(2). Therefore, the question of selecting a good initial approximation, preserving properties of nonlinearity, arises. Depending on the parameters of the equation, this difficulty is overcome by appropriate choice of initial approximations, which are accepted by the asymptotic formula established above. We produced the numerical calculations on the basis of the obtained qualitative properties. The numerical results show fast convergence of the iterative process to the solution of the Cauchy problem (1)-(2) due to the successful choice of the initial approximation. Below we give some results of numerical experiments for different values of the numerical parameters.

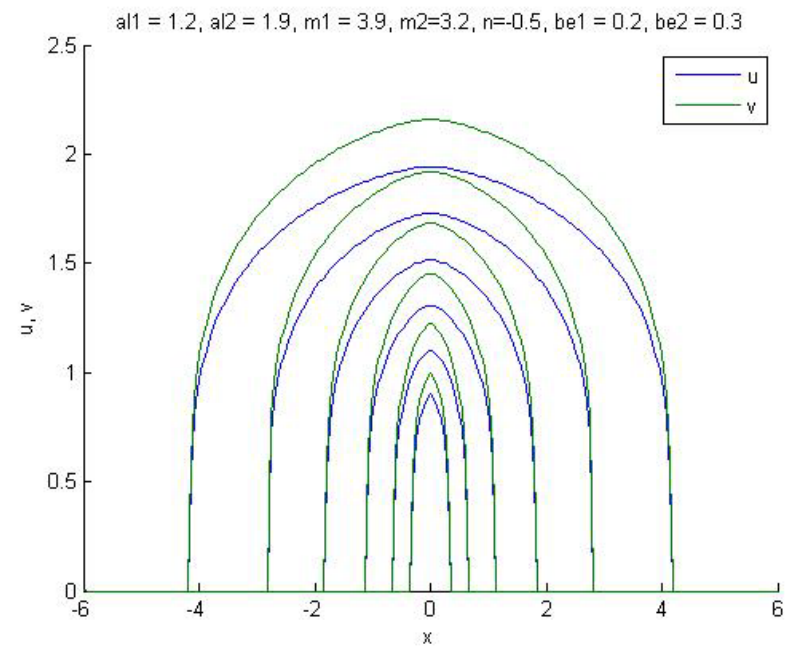

Figure 1. Slow diffusion. Case $\alpha_{1}>1, \alpha_{2}>1, \mathrm{n}<0$.

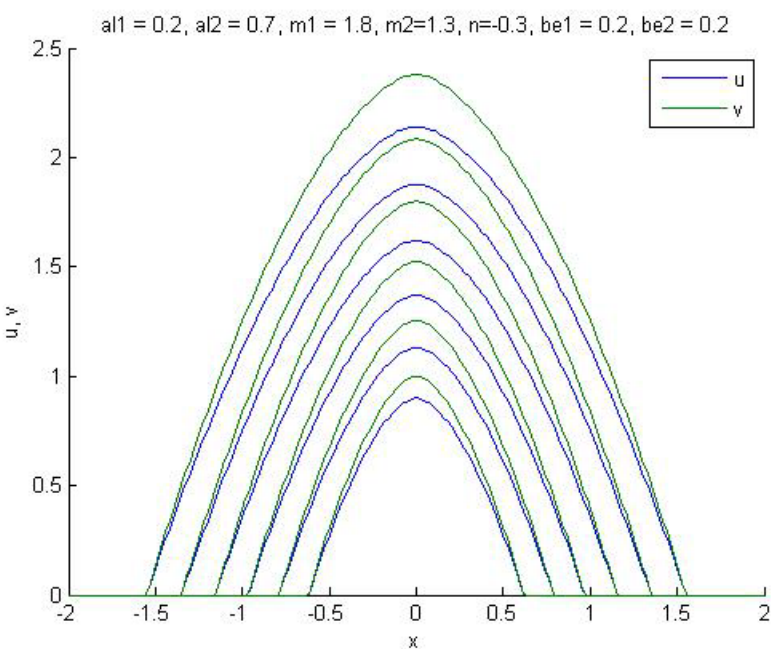

Figure 2. Slow diffusion. Case $\alpha_{1}<1, \alpha_{2}<1, \mathrm{n}<0$.

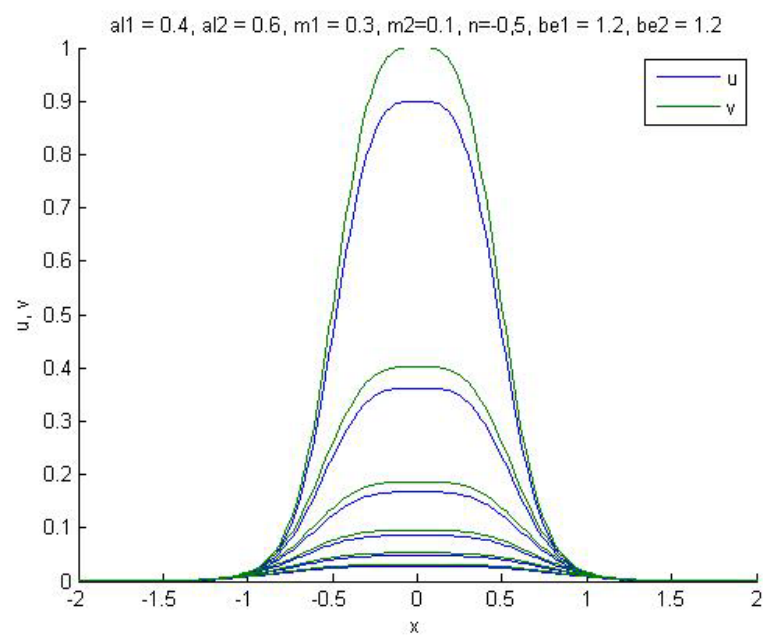

Figure 3. Fast diffusion. Case $\alpha_{1}<1, \alpha_{2}<1, \mathrm{n}<0$. 


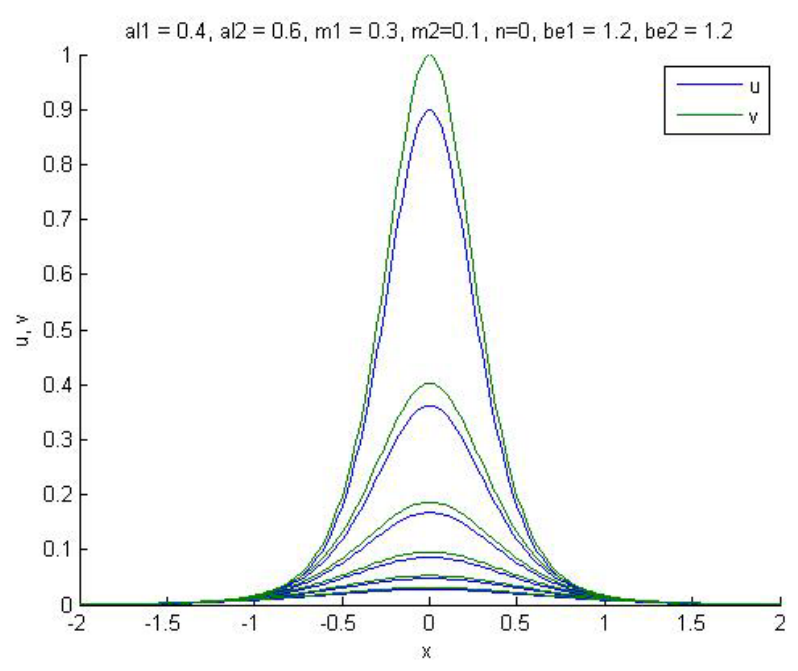

Figure 4. Fast diffusion. Case $\alpha_{1}<1, \alpha_{2}<1, \mathrm{n}=0$.

As one can see from Figures 1-4, the results of numerical experiments are close enough to the self-similar solutions. The figure 1 and figure 2 show a compactly supported solution of the problem (1)-(2). In the figure 3 and figure 4 shown the solution of the problem (1)-(2) vanishing at infinity.

\section{Conclusions}

The property a FSPD of the Cauchy problem for a cross diffusion parabolic system not in divergence form with a source and a variable density based on comparison method is considered. An asymptotic behavior of self-similar solutions both for slow diffusion and fast diffusion cases are established. It is shown that coefficients of main terms of asymptotic of solution satisfy to some system of a nonlinear algebraic equation.

Results of computational experiments show that the self-similar solutions are very appropriate approximation. And the iterative method based on the Picard's method is effective for the solution of nonlinear problems and retains the nonlinear effects, if we will use as an initial approximation the solutions of self-similar equations, constructed by the method of nonlinear splitting and by the method of standard equation [7-9].

\section{REFERENCES}

[1] M.X. Wang, C.H. Xie. A degenerate strongly coupled quasilinear parabolic system not in divergence form, $\mathrm{Z}$. Angew. Math. Phys., 55, 741-755, 2004.

[2] Haihua Lu. Global existence and blow-up analysis for some degenerate and quasilinear parabolic systems, Electronic Journal of Qualitative Theory of Differential Equations, No. 49, 1-14, 2009.

[3] W. Zhou, Z. Yao. Cauchy problem for a degenerate parabolic equation with non-divergence form, Acta. Mathematica Scienta, 30 B, No.5, 1679-1686, 2010.

[4] Chunhua Jin, Jingxue Yin. Self-similar solutions for a class of non-divergence form equations, Nonlinear Differ. Equ. Appl. Nodea, Vol. 20, Issue 3, 873-893, 2013.

[5] J.R. Raimbekov. The Properties of the Solutions for Cauchy Problem of Nonlinear Parabolic Equations in Non-Divergent Form with Density, Journal of Siberian Federal University. Mathematics and Physics, 8 (2), 192-200, 2015.

[6] A.A. Samarskii, V.A. Galaktionov, S.P. Kurdyumov and A.P. Mikhailov. Blow-Up in Quasilinear Parabolic Equations, Berlin, 4, Walter de Grueter, p. 535, 1995.

[7] M. Aripov. Method of the Standard Equation for the Solution of the Nonlinear Value Problem, Fan, Tashkent, 137 p., 1988.

[8] M. Aripov, Sh.A. Sadullaeva. Qualitative Properties of Solutions of a Doubly Nonlinear Reaction Diffusion System with a Source, Journal of Applied Mathematics and Physics, 3, 1090-1099, 2015.

[9] M. Aripov, Sh.A. Sadullaeva. An asymptotic analysis of a self-similar solution for the double nonlinear reaction-diffusion system, J. Nanosystems: physics, chemistry, mathematics, 6 (6), 1-10, 2015.

[10] A.V. Martynenko, A.F. Tedeev. The Cauchy Problem for a Quasilinear Parabolic Equation with a Source and Inhomogeneous Density, Computational Mathematics and Mathematical Physics, 47, 238-248, 2007.

[11] A.V. Martinenko, V.N. Shramenko. Estimate of solutions of the Cauchy Problem near the Time of Exacerbation for a Quasilinear Parabolic Equation with a Source and a Variable Density, Nonlinear Boundary Value Problems, 20, 104-115, 2010.

[12] M. Aripov, Z. Rakhmonov. Estimates and Asymptotic of Self-similar Solutions to a Nonlinear Filtration Problem with Variable Density and Nonlocal Boundary Conditions, Universal Journal of Computational Mathematics, 4(1), 1-5, 2016.

[13] Sh.A. Sadullaeva. Numerical Investigation of Solutions to a Reaction-Diffusion System with Variable Density, Journal of Siberian Federal University. Mathematics and Physics, V. 9 (1), 90-101, 2016. 